Original Research Paper

\title{
Implementation of Geodesic Active Contour Approach for Pigment Spots Segmentation on the Iris Surface
}

\author{
${ }^{1}$ Mustafa Man, ${ }^{2}$ Mohamad Faizal Ab Jabal, ${ }^{3}$ Mohd Shafry Mohd Rahim and ${ }^{4}$ Illiasaak Ahmad \\ ${ }^{I}$ School of Informatics and Applied Mathematics, University Malaysia Terengganu, Kuala Terengganu, Terengganu, Malaysia \\ ${ }^{2}$ Digital Graphic and Media (DiGM) Research Group, Faculty of Computer and Mathematical Sciences, \\ University Technology MARA Johor Branch, Pasir Gudang Campus, Johor, Malaysia \\ ${ }^{3}$ UTM ViCubeLab Research Group, Faculty of Computing, University Technology Malaysia, Johor, Malaysia \\ ${ }^{4}$ Department of Computer Science, Faculty of Computer and Mathematical Sciences, \\ University Technology MARA Kedah Branch, Merbok Campus, Kedah, Malaysia
}

Article history

Received: 12-05-2016

Revised: 24-11-2016

Accepted: 25-12-2016

Corresponding Author: Mohamad Faizal Ab Jabal Digital Graphic and Media (DiGM) Research Group, Faculty of Computer and Mathematical Sciences, University Technology MARA Johor Branch, Pasir Gudang Campus, Johor, Malaysia Email: m.faizal@johor.uitm.edu.my

\begin{abstract}
The aim of this paper is to study the effectiveness of geodesic active contour approach to segment the pigment spots on iris surface. Pigment spot is an abnormal marker that appears on the iris surface, which may lead to eye diseases. The multi appearance, scattered location and dynamic shape of the pigment spots on the iris surface make them very difficult to detect using automatic segmentation method. Therefore, this paper presents the preliminary works that observe the efficiency and accuracy of geodesic active contour approach to automatically segment the pigment spots on iris surface. Miles database and "lu_weiffeb_2006" dataset are used in this study. The results of the conducted experiment show that geodesic active contour approach able to correctly and accurately detect up to $77 \%$ and $90 \%$ of the pigment spots in Miles database and "lu_wei_feb_2006" dataset, respectively.
\end{abstract}

Keywords: Geodesic Active Contour, Iris Segmentation, Iris Surface, Miles Research Database, Pigment Spot

\section{Introduction}

Iris recognition system can be applied to various applications. The used of iris recognition system in medical field, known as Ophthalmology, has been well accepted by health practitioners. Ophthalmology is the area of study that focuses on the health care of the iris, retina and eyes (Preethi and Jayanthi, 2014). The existence of pigment spots on the iris surface, as shown in Fig. 1.1, may indicate the possibility of eye diseases (Preethi and Jayanthi, 2014). However, due to the dynamic form of the pigment spots on the iris surface make it very difficult to be segmented automatically using iris recognition system.

Figure 1.2 shows challenges in iris segmentation, which can be categorized into two: Common and rare issues. The common issue such as occlusion, motionblurred and off-angled are well study by various researchers (Jillela and Ross, 2013) particularly in biometric authentication systems. However, the outlier issue is a rare case in authentication system, but it is a common situation can be found in ophthalmology field
(Preethi and Jayanthi, 2014). The outlier situation has been defined as the acquired eye image with abnormalities in the shape of the iris as shown in Fig. 1.1. These abnormalities may interrupt the recognition process of the iris (Jillela and Ross, 2013). Based on the outlier definition and considering the pigment spots description, this paper has extended the outlier definition to the iris image that contains the pigment spots on the iris surface, which is also possible to interrupt the recognition process (Man et al., 2016).

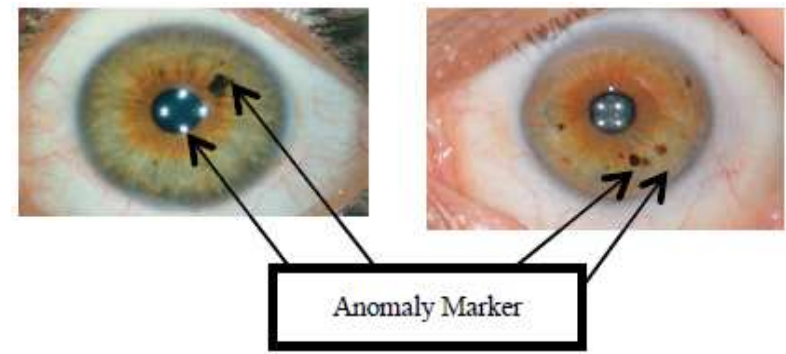

Fig. 1.1. Outlier's situation (pigment spots) on the iris surface 


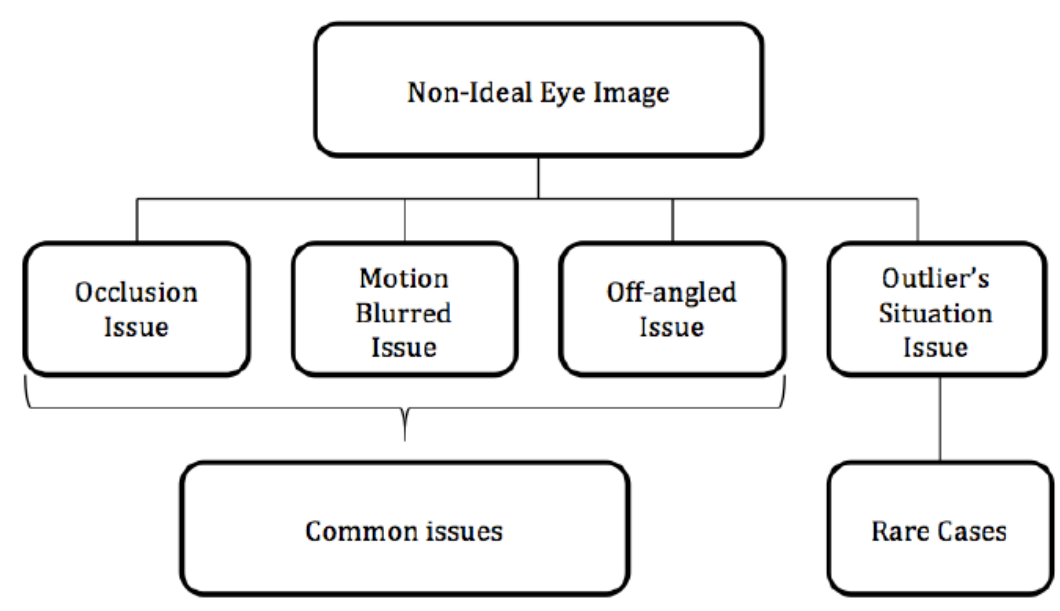

Fig. 1.2. Challenges in iris segmentation

The main purpose of segmenting the pigment spot on the iris surface is to detect abnormality symptom or activity on the iris surface that may indicate a possibility of eye diseases. The dynamic forms of the pigment spot make it rather hard to be detected automatically. This study applied the geodesic active contour approach to detect and segment the pigment spots. The main idea to use this approach is to evaluate the efficiency and accuracy of geodesic active contour in segmenting the multi-objects in images. The multi-objects in this study are referred to pigment spots. Based on the method proposed by the author applied the geodesic active contour approach to segment the Kaposi Sarcoma in medical MRI image (Sandhu et al., 2008). For education and research purposes, the author shared the program source code online and is used in this study. Few modifications of the program were made to suit the objective of this study.

The structure of the paper is organized as follows. Presentation on the related work will be in literature review section. Methodology section provides an overview of the dataset used for the experiment. The method of the geodesic active contour approach also been explained in these section. The segmentation performance is reported in testing section. Conclusion section concludes the paper and the future work.

\section{Literature Review}

\section{Multi-Object Image Segmentation Approaches}

Table 2.1 shows several prominent multi-object image segmentation approaches targeted for medical purposes. It can be said that these approaches are highly accurate in segmenting the region of interest of multi-object in an image. Nevertheless, the processing time to perform the detection is still unacceptable. For instance, Chen et al. (2012; Kéchichian et al., 2011) took around $300 \mathrm{sec}$. and $720 \mathrm{sec}$. to correctly detect the region of interest in an image, respectively. It was active contour approach that stands out in terms of processing time as proposed by Lucas et al. (2012; Sandhu et al. (2008). They not only managed to accurately segment region of interest but also speed up the processing time.

\section{Iris Boundary Segmentation Approaches}

Back in 1994, Daugman have introduced Integrodifferential operator to automate the segmentation of the iris region in eye images (Jillela and Ross, 2013). However, the approach does not consider the eyelashes and eyelids. This allows other researchers to contribute into the field. Wildes proposed circular Hough transform to accurately segment the iris region on the eye image (Jillela and Ross, 2013).

Table 2.1. Multi-object segmentation approaches

\begin{tabular}{|c|c|c|}
\hline $\begin{array}{l}\text { Segmentation approach } \\
\text { (Author/Year) }\end{array}$ & $\begin{array}{l}* \text { Accuracy } \\
\text { rate }\end{array}$ & $\begin{array}{l}\text { Processing } \\
\text { time }\end{array}$ \\
\hline $\begin{array}{l}\text { Clustering and graph cuts } \\
\text { (Kéchichian et al., 2011; } \\
\text { Beichel et al., 2012; } \\
\text { Hernandez-Vela et al., 2012) }\end{array}$ & High & $\begin{array}{l}\text { } 720 \text { sec. } \\
\text { (Kéchichian et al., } \\
\text { 2011) }\end{array}$ \\
\hline $\begin{array}{l}\text { Graph cuts and oriented } \\
\text { active appearance models } \\
\text { (Chen et al., 2012) }\end{array}$ & High & $\sim 300$ sec. \\
\hline $\begin{array}{l}\text { Mesh-coupling algorithm } \\
\text { (Dagmar, 2015) }\end{array}$ & $* * \mathrm{NA}$ & $* * N A$ \\
\hline Visual patch (Li et al., 2015) & High & $* * N A$ \\
\hline $\begin{array}{l}\text { Active contour (Gao et al., } \\
\text { 2011) }\end{array}$ & High & $\sim 45 \mathrm{sec}$ \\
\hline $\begin{array}{l}\text { Geometric active contour } \\
\text { with energy model } \\
\text { (Sandhu et al., 2008) }\end{array}$ & High & $* *$ NA \\
\hline $\begin{array}{l}\text { Geodesic active contours } \\
\text { (Lucas et al., 2012) }\end{array}$ & High & $\sim<10 \mathrm{sec}$ \\
\hline
\end{tabular}


Later, with a slight modification of the circular Hough transform algorithm Masek employed the revised Hough transform algorithm to extract the iris region. Result from Masek experiment shown that the improved algorithm was accurately extracted iris region with the accuracy of $98.6 \%$ (Jillela and Ross, 2013). Although the result has shown an improvement towards the approach, the reliability of the eye images used in that experiment has been questions. Bowyer et al. (2013) stated the eye image from CASIA database (CASIA version 1) is not reliable to be used as a primary dataset because the image has been edited to get a perfect circle on the pupil and iris. Moreover, the pupil color has been modified to become darker than a normal pupil. It is to increase the color intensity for a reason to ease the pupil region detection during segmentation process. Therefore, every significant approach that been developed and tested based on the CASIA database will achieve high accuracy rate. However, when the approach was tested against with a secondary dataset the outcome shows a negative result as presented in Fig. 2.1.

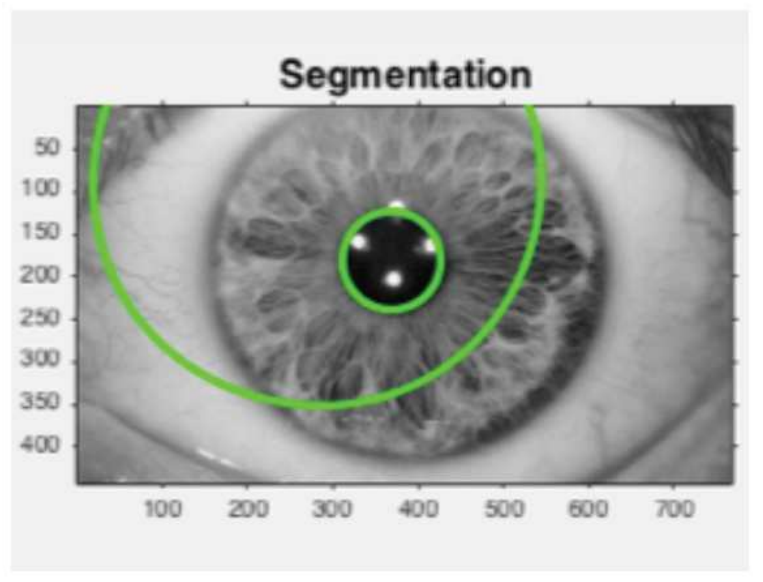

Fig. 2.1. Inaccurate segmentation on iris boundary

Table 2.2. Iris Boundary Segmentation Approaches (Jillela and Ross, 2013)

\begin{tabular}{ll}
\hline Author(s) & Approach \\
\hline Shah and Ross (2003) & Geodesic active contour \\
Roy et al. $(2007)$ & Variational level sets \\
Daugman (2003) & Fourier-based approximation \\
Schuckers et al. (2007) & Active shape models \\
Pundlik et al. $(2008)$ & Graph cuts \\
Zuo and Schmid (2010) & Ellipse fitting \\
He et al. (2013) & Pulling and pushing model \\
Du et al. $(2014)$ & Scale invariants feature transform \\
Masek et al. $(2015 ;$ & Circular Hough transform \\
Lim et al., 2014; & \\
Huang et al., $1990 ;$ & \\
Yuan et al. (2005) & Edge detection and Hough transform \\
Huang et al. $(1990)$ & Phase congruency and Hough \\
& transform \\
\hline
\end{tabular}

Based on available documented literatures, Jillela and Ross (2013) had reported there is various iris segmentation approaches have been proposed to deal with the non-ideal eye image such as off-angled, occlusion and blurry motion situation as summarizes in Table 2.2. However, it is very rare to find documented publications on pigment spots. This is due to the fact that the pigment spot is not a main concern in the iris-based authentication system when compared to healthcare related systems like ophthalmology.

\section{Methodology}

\section{Dataset}

Figure 3.1 shows the sample of the iris image that was used in this study. It is known as Miles Research database prepared by Miles Research Company. The images have been acquired by using PV320C. Size of the image is $1749 \times 1184$ pixels in 256 dpi resolutions. The images have been stored in Joint Photographic Experts Group (jpeg) file format. The color space is 24-bits RGB (Johnson, 1984; Miles Research, 2013). The image has been filtered manually into two categories, which are normal iris image and anomaly iris image. Since the objective of this work is to segment the pigment spots on the iris surface only the anomaly category was used.

In addition, Fig. 3.2 is another dataset named "lu_weiffeb_2006" was also used in order to have different type of images and ultimately study the efficiency geodesic active contour approach. This dataset was prepared by Russell et al. (2008) from Computer Science and Artificial Intelligence Laboratory, Massachusetts Institute of Technology (MIT), Cambridge, USA. Unfortunately, there is no detail information regarding the equipment used or standard operating procedure (S.O.P) on how the images were acquired (Russell et al., 2008).

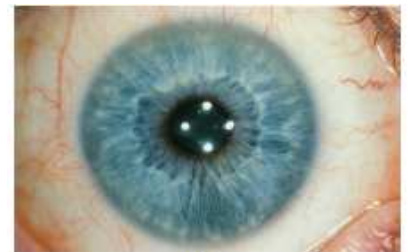

(a)

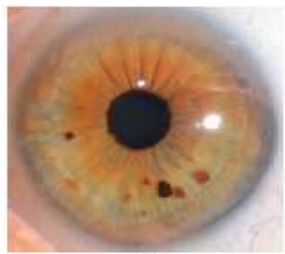

(b)
Fig. 3.1.Miles Research iris database: (a) Normal iris image, (b) An iris image contains anomaly marker (pigment spots) on the iris surface

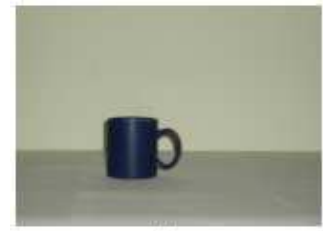

(a)

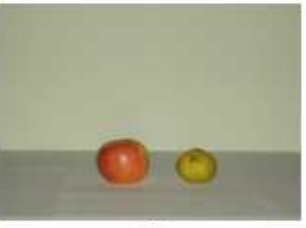

(b)
Fig. 3.2. "lu_wei_feb_2006" dataset, (a) Single object, (b) Multi-object 


\section{Pre-Processing}

The first phase in this study is to segment the iris position from the eye image. Circular Hough transforms was deployed, where the iris is the ROI in this study. The approach is used because the ability of the approach to approximate the center and radius coordinates of the pupil and iris regions when the numbers of points that fall on the parameter are known (Rai and Yadav, 2014). The process starts with the image conversion from RGB to gray scale image. Then canny edge detection is applied in order to find the edges. This process can be presented as arithmetical equation as shown in (1) and (2):

$x=x_{c}+r * \cos \theta$

$y=y_{c}+r * \sin \theta$

where, $r$ is a radius and $x_{c}, y_{c}$ is a center coordinate of the circle. While $\theta$ is a degree of the angle when it sweeps through the full $360^{\circ}$ range, the points $(x, y)$ will trace the boundary of the circle. Then for all edge points $\left(x_{i}\right.$, $\left.y_{i}\right), I=1,2 \ldots n$ Hough transform is applied in order to segment the pupil and iris region. The arithmetic equivalent of Hough transform is shown in (3) and (4):

$H\left(x_{c}, y_{c}, r\right)=\sum_{i=1}^{n} h\left(x_{i}, y_{i}, x_{c}, y_{c}, r\right)$

$h\left(x_{i}, y_{i}, x_{c}, y_{c}, r\right)=\left\{\begin{array}{l}1, \text { if } g\left(x_{i}, y_{i}, x_{c}, y_{c}, r\right)=0 \\ 0, \text { otherwise }\end{array}\right\}$

where, $g\left(x_{i}, y_{i}, x_{c}, y_{c}, r\right)=\left(x_{i}^{2}-x_{c}^{2}\right)+\left(y_{i}^{2}-y_{c}^{2}\right)-r^{2}$. The three coordinates $\left(x_{c}, y_{c}, r\right)$ for which $H\left(x_{c}, y_{c}, r\right)$ is highest will be the coordinate of the center and radius of the circle. Figure 3.3 shows the output of the process.
On the other hand, it is not necessary for "lu_weiffeb_2006" dataset to be extracted into the ROI form, because the obtained image is ready for segmentation process. However, the images need to go through the gray scale and masking processes in the preprocessing phase.

\section{Object Segmentation Process}

The second phase of this work was to segment the pigment spots on the extracted ROI images using geodesic active contour approach as shown in Fig. 3.4. A modified version of, (Sandhu et al., 2008) source code was used. The first modification was took place on the image acquisition, so the program is able to receive the visible light and high resolution of the image instead of gray scale image and $32 \times 32$ resolution of image. Next modification is on the iteration, where the original is 800 and has been changed to 500 loops.

The first step is to assume $I$ as the ROI image consists of the object and background. In this case the object is the pigment spots and the background is the iris surface. The process started by calculating the similarity metrics in the image to portray the object or the foreground. Hence level set algorithm has been used to proceed with the process. Let the curve $C$ represent a zero-level set to a signed distance function $\phi: \mathfrak{R}^{2} \rightarrow \mathfrak{R}$ and if $\phi<0$ it will be indicated the inside of $C$, otherwise it is outside of the $C$. This indication is presenting the object or background in the ROI image, where the inside of the curve $C$ matched that means the object, else is a background. A more thorough discussion of the equation can be found in (Sandhu et al., 2008).

Next, the values of the metrics were used to threshold the image where the curve $C$ on the ROI image is a boundary that separating between the object and the background. Finally, the boundary detection program is executed to draw the outline of the boundary to show the segment object found in the ROI as presented in Fig. 3.5 and 3.6.

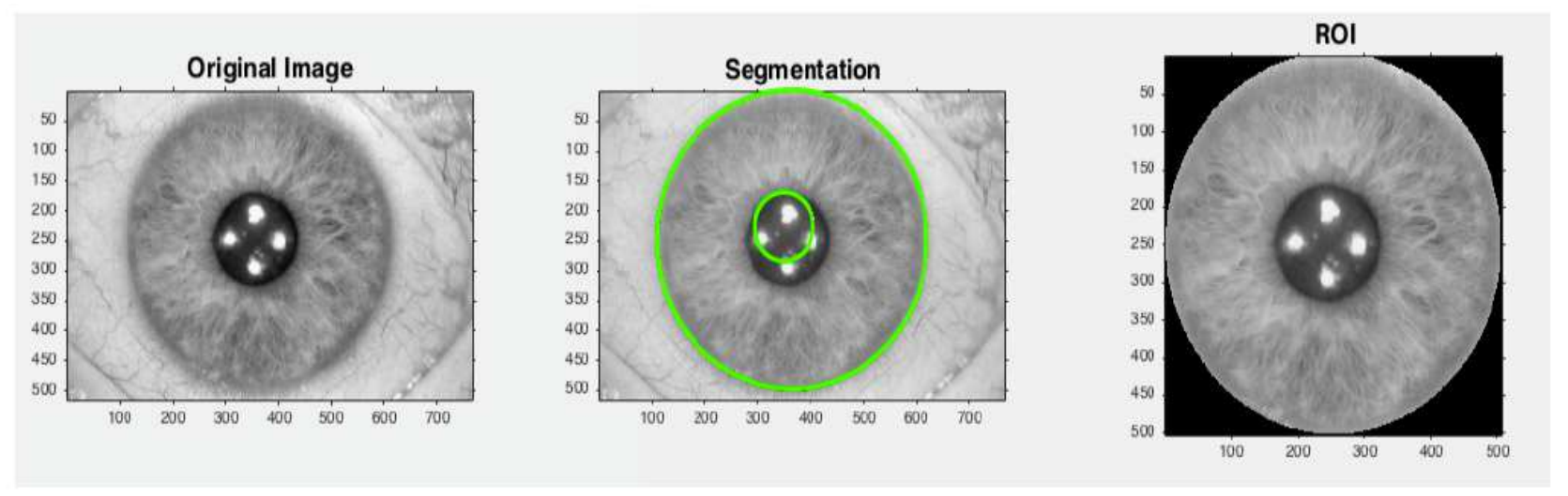

Fig. 3.3. Pupil and iris region segmentation 


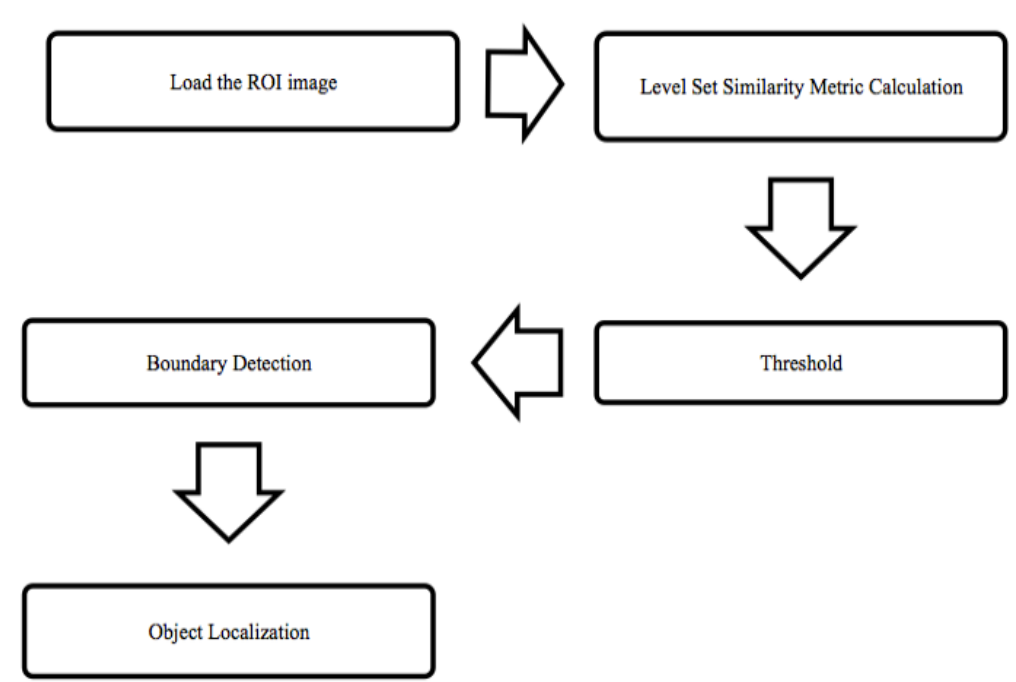

Fig. 3.4. Object segmentation method (Adapted from Sandhu et al., 2008)

\section{Testing}

\section{Setup}

The experiment was conducted based on the following hardware and software specifications; $2.3 \mathrm{GHz}$ processor, $8 \mathrm{~GB}$ of memory with speed of $1333 \mathrm{MHz}$, Intel HD graphics $3000512 \mathrm{MB}$, OS X version 10.11 as a platform, MATLAB R2014B. Non-iris images from "lu_weiffeb_2006" and Sandhu et al. (2008) were used in the first experiment. In the second experiment Miles's iris dataset was applied to the test program. In both experiments the test program must perform single and multi-object segmentation.

\section{Result and Validation}

Figure 3.5 and 3.6 shows the result of the first experiment that has been conducted using non-iris images. It can be clearly seen that Sandhu's approach had successfully segmented a single object. It also correctly detects the multi-object in the image. This study believes that Sandhu's approach should able to segment the pigment spots on the iris surface. To prove our claim, we fed in iris images from Miles database to the test program.

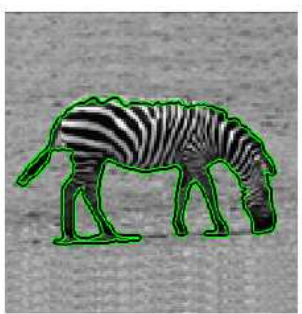

(a)

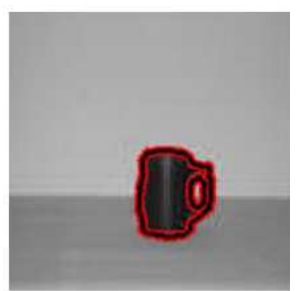

(b)
Fig. 3.5. Example of segmentation result of a single object, (a) from Sandhu et al. (2008) dataset, (b) from lu_wei_feb_2006" dataset
Figure 4.1 presents the sample of correct iris segmentation results while Fig. 4.2 presents the sample of incorrect segmentation results of Miles database. In general, it can be said that the objects was successfully segmented. Although the accuracy rate achieved is only around $77 \%$ (Table 4.1), it proves that Sandhu's approach can be applied to segment the pigment spots on iris surface. There are few factors that contribute to low accuracy rate.

First, after further investigation to the images in Miles database we found that the boundary detection process was influence by the color and illumination intensity of the pigment spots. Second, the incorrect threshold values have been produced in order to create the mask.

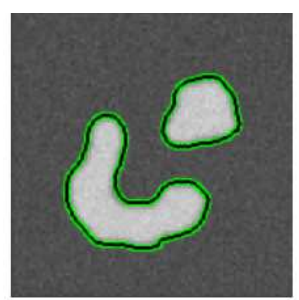

(a)

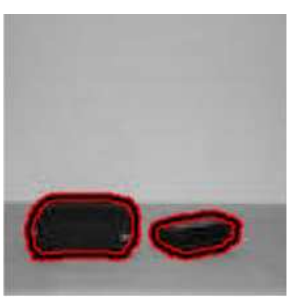

(b)
Fig. 3.6. Example of segmentation result of multi-object, (a) from Sandhu et al. (2008) dataset, (b) from lu_weiffeb_2006

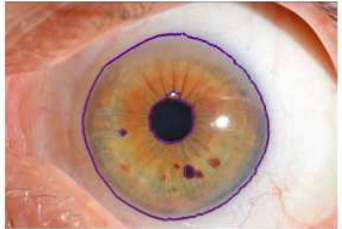

(a)

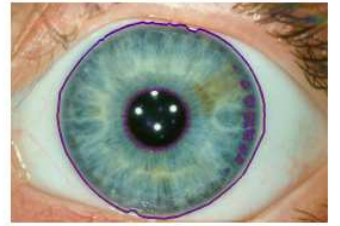

(b)
Fig. 4.1. Example of correct segmentation result using Miles database 


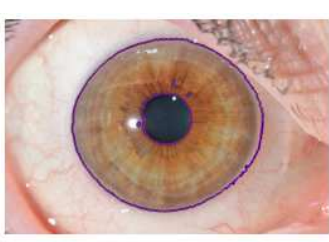

(a)

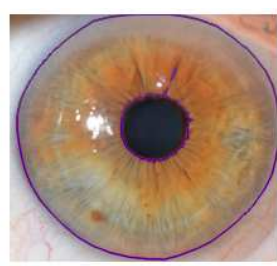

(b)
Fig. 4.2.Example of incorrect segmentation result using Miles database

We also found out that the pigment spots is slightly difficult to detect when the color of the pigment spots is nearly the same as the color of the iris surface. A darker color of pigment spots is desirable for this purpose. Furthermore, light reflection on the pupil area and iris surface also influence the boundary detection process.

The average of the execution time for the program to run all the processes for every single image from Miles database is between 4-7 min. While for the "lu wei feb 2006" dataset its take around 1-3 min. The slight difference on the execution time is because of the resolution size of the images. Moreover the iteration for the boundary detection has been set around 500 loops. Therefore the execution time for Miles database is slower than the "lu wei feb 2006" dataset.

Based on the experiment, the generated output from the execution program for the boundary detection in order to segment the pigment spots was fairly performed but in certain cases it was imprecise as shown in Fig. 4.3. Overall, the geodesic active contour has a potential and reliable to be applied towards the pigment spots segmentation on the iris surface or multi-objects segmentation in the image.

There are, however, several issues that must be addressed in which we believed contribute to low execution performance and accuracy rate. First, the used of boundary feature detection to segment objects by seeking the similarity metrics on the image is not suitable to be applied on the iris image. It is because the iris surface is not a plain surface as images from "lu_weiffeb_2006" dataset. The iris surface consists of the iris various patterns and colors. If the existence of the pigment spots on the iris surface had a similar shape or pattern and color with the iris surface, then the approach is easy to miscalculate the similarity metrics, when the process of calculating is acquired the values or data from the grey scale image. It is because the program will convert from full RGB into the grey scale image. The conversion process eliminates the important information regarding the pigment spots especially the color of the marker. Therefore, it will produce a wrong direction to the program to segment the pigment spots.

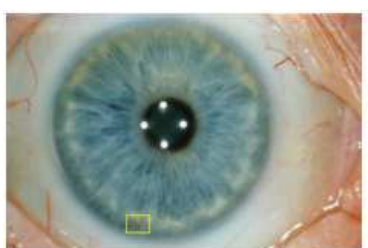

a) Manually segmented

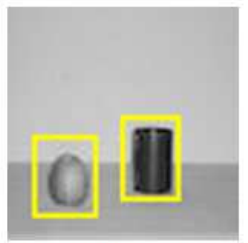

c) Manually Segmented

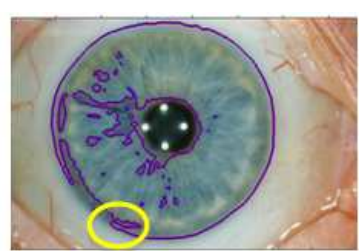

b) Segmented by the program

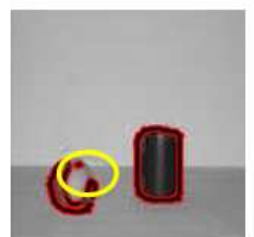

d) Segmented by the program
Fig. 4.3. Example of imprecise segmentation, (a) and (b) images from Miles database; (c) and (d) images from "lu_wei_feb_2006"

Table 4.1. The segmentation accuracy rate

\begin{tabular}{lll}
\hline Database/dataset & $(\%)$ & $\begin{array}{l}\text { Processing time } \\
\text { minute/image } \\
\text { (Average) }\end{array}$ \\
\hline Miles Database & 77 & $4-7$ \\
"lu_wei_feb_2006" dataset & 89 & $1-3$ \\
\hline
\end{tabular}

Although the image from "lu wei feb 2006" dataset was obtained with the plain background, still the approach is able to miscalculate the similarity metrics and produced imprecise segmentation output. The reason is the illumination of the light or reflection was generated high intensity of the color on the object. Therefore, when the image was converted into the grey scale, it was found that the spot with the high intensity on the object is presented as white. It will give a different meaning to the program when the grey scale image is convert into the binary image, where the white color will be represent as value 1. At the same time the value of the object that should be segmented will be represent as 0 . Hence the boundary line will be drawn on the objects that represent 0 in the image. As a result, imprecise segmentation to the object will be generated.

Second, the execution time for each image from both databases to be processed could be considers as slow processing time. The calculation process influenced the resolution size of the image during seeking the similarity metrics. The program will search for the similarity point all around the image. Next the higher resolution size of the image, the slower execution time will be produced from the program to generate the segmentation output.

Although the result from the conducted experiment does not show the robust achievement regarding the detection and segmentation, but it is enough to prove that the geodesic active contour approach is reliable to be applied for pigment spots segmentation on the iris surface. 
Most publications regarding the geodesic active contour approach focused on segmenting big objects in the image compared with the pigment spots on the iris surface, which is smaller in size, exhibits various patterns and colors of the object to be detected. Therefore in detecting and segmenting the smaller objects on the various patterns and colors background is much challenging compared with the ordinary conducted lab experiment with the lab ideal image or database set.

\section{Conclusion}

This paper presented the application of geodesic active contour approach to detect and segment the pigment spots on iris surface. Our experiments have shown that it can be applied not only to segment multiobject on various shapes but also with different sizes. A modification of (Sandhu et al., 2008) geodesic active contour approach has also shown an improvement in segmentation accuracy rate.

\section{Future Work}

Further research can be conducted on the approach in which it can be applied to segment micro objects on the various backgrounds in the image. Moreover, it is also possible to use geodesic active contour with current boundary feature algorithm to improve the segmentation method. As a result of this marriage the segmentation will be more precise compare and increase the segmentation accuracy rate.

\section{Acknowledgement}

This work would like to thank you to all colleagues at University Malaysia Terengganu (UMT) to fund this publication, Digital Graphic \& Media (DiGM) Research Group, University Technology MARA (UiTM) Johor branch, ViCubeLab Research Group, University Technology Malaysia (UTM) and University Technology MARA (UiTM) Kedah branch for the ideas and supports.

\section{Author's Contributions}

Mustafa Man: Contributed in organizing the experiments and data analysis. Co-supervisor of this study and as a funder for this study.

Mohamad Faizal Ab Jabal: Participated in all fundamental and literature study. Conducted all experiments and data analysis. Contributed to the writing of the manuscript. Researcher of this study.

Mohd Shafry Mohd Rahim: Contributed on research plan and organized the study. Main supervisor of this study.

Illiasaak Ahmad: Participated as proof reader and editor.

\section{Ethics}

The authors confirm that this manuscript has not been published elsewhere and that no ethical issues are involved.

\section{References}

Beichel, R., A. Bornik, C. Bauer and E. Sorantin, 2012. Liver segmentation in contrast enhanced CT data using graph cuts and interactive 3D segmentation refinement methods. Med. Phys., 39: 1361-1373. DOI: $10.1118 / 1.3682171$

Bowyer, K.W, Hollingsworth, K.P, Flynn, P.J. 2013. A Survey of Iris Biometrics Research: 2008-2010. In: Handbook of Iris Recognition, Burge, M.J. and K. Bowyer (Eds.), Springer Science and Business Media, London, ISBN-10: 1447144023, pp: 15-54.

Chen, X., J.K. Udupa, U. Bagci, Y. Zhuge and J. Yao, 2012. Medical image segmentation by combining graph cuts and oriented active appearance models. IEEE Trans. Image Process., 21: 2035-2046. DOI: $10.1109 /$ TIP.2012.2186306

Dagmar, K., 2015. Multi-Object Segmentation of Joints. Deformable Meshes for Medical Image Segmentation: Accurate Automatic Segmentation of Anatomical Structures, Dagmar, K. (Ed.), Springer, Wiesbaden, ISBN-10: 3658070153, pp: 117-129.

Daugman, J., 2003. The importance of being random: Statistical principles of iris recognition. Patt. Recognit., 36: 279-291.

Du, T., Z. Han, G. Zhou and B. Roizman, 2014. Patterns of accumulation of miRNAs encoded by herpes simplex virus during productive infection, latency and on reactivation.

Gao, Y., A. Tannenbaum and R. Kikinis, 2011. Simultaneous Multi-Object Segmentation using Local Robust Statistics and Contour Interaction. Medical Computer Vision: Recognition Techniques and Applications in Medical Imaging, Menze, B., G. Langs, Z. Tu and A. Criminisi (Eds.), Springer, Heidelberg, ISBN-10: 3642184219, pp: 195-203.

He, G., D. Dhar, H. Nakagawa, J. Font-Burgada and H. Ogata et al., 2013 Identification of liver cancer progenitors whose malignant progression depends on autocrine IL-6 signaling. Cell, 155: 384-396. DOI: $10.1016 /$ j.cell.2013.09.031

Hernandez-Vela, A., N. Zlateva, A. Marinov, M. Reyes and P. Radeva et al., 2012. Graph cuts optimization for multi-limb human segmentation in depth maps. Proceedings of the IEEE Conference on Computer Vision and Pattern Recognition, Jun. 16-21, IEEE Xplore Press, pp: 726-732. DOI: $10.1109 /$ CVPR.2012.6247742

Huang, T.T., N.C. Groves and G. Belt, 1990. Boundarylayer flow on an axisymmetric body with an inflected stern. 
Jillela, R. and A.A. Ross, 2013. Methods for Iris Segmentation. In: Handbook of Iris Recognition, Burge, M.J. and K. Bowyer (Eds.), Springer Science and Business Media, London, ISBN-10: 1447144023, pp: $15-54$.

Johnson, D., 1984. What the Eye Reveals: An Introduction to the Rayid Method of Iris Interpretation. 1st Edn., Rayid Model Publications, Goleta, CA, ISBN-10: 0917197011.

Kéchichian, R., S. Valette, M. Desvignes and R. Prost, 2011. Efficient multi-object segmentation of 3D medical images using clustering and graph cuts. Proceedings of the 18th IEEE International Conference on Image Processing, Sept. 11-14, IEEE Xplore Press, pp: 2149-2152. DOI: 10.1109/ICIP.2011.6116036

Li, P., J. Feng, Q. Bu, F. Liu and H. Wang, 2015. MultiObject Segmentation for Abdominal CT Image Based on Visual Patch Classification. In: Computer Vision, Zha, H., X. Chen, L. Wang and Q. Miao (Eds), Springer, Heidelberg, ISBN-10: 3662485702 , pp: 130-138.

Lim, E.T., P. Würtz, A.S. Havulinna, P. Palta and T. Tukiainen et al., 2014. Distribution and medical impact of loss-of-function variants in the finnish founder population. PLoS Genet, 10: e1004494e1004494. DOI: 10.1371/journal.pgen.1004494

Lucas, B.C., M. Kazhdan and R.H. Taylor, 2012. MultiObject Geodesic Active Contours (MOGAC): A parallel sparse-field algorithm for image segmentation. Department of Computer Science, Johns Hopkins University.

Man, M., M.F.A. Jabal and M.S.M. Rahim, 2016. A Comparative Study on Hough Transform Segmentation Approach for Outlier Iris Image. In: Information Science and Application, Kim, K.J. (Ed.), Springer, Heidelberg, ISBN-10: 3662465787 , pp: 409-419.

Masek, P., K. Worden, Y. Aso, G.M. Rubin and A.C. Keene, 2015. A dopamine-modulated neural circuit regulating aversive taste memory in Drosophila. Curr. Biol., 25: 1535-1541. DOI: 10.1016/j.cub.2015.04.027
Miles Research, 2013. Miles research iris database.

Preethi, D.M.D. and V.E. Jayanthi, 2014. Ocular disease diagnosis based on LBP and Gabor filter. Int. J. Sci. Eng. Res., 5: 297-304.

Pundlik, S.J., D.L. Woodard and S.T. Birchfield, 2008. Non-ideal iris segmentation using graph cuts. Proceedings of the IEEE Computer Society Conference on Computer Vision and Pattern Recognition Workshops, Jun. 23-28, IEEE Xplore Press, Anchorage, AK, pp: 1-6. DOI: $10.1109 /$ CVPRW.2008.4563108

Rai, H. and A. Yadav, 2014. Iris recognition using combined support vector machine and Hamming distance approach. Expert Syst. Applic., 41: 588-593. DOI: 10.1016/j.eswa.2013.07.083

Roy, S., T. Lane, C. Allen, A.D. Aragon and M. WernerWashburne, 2007. A hidden-state markov model for cell population deconvolution. 13: 1749-1774. DOI: $10.1089 / \mathrm{cmb} .2006 .13 .1749$

Russell, B.C., A. Torralba, K.P. Murphy and W.T. Freeman, 2008. LabelMe: A database and web-based tool for image annotation. Int. J. Comput. Vis., 77: 157-173. DOI: 10.1007/s11263-007-0090-8

Sandhu, R., T. Georgiou and A. Tannenbaum, 2008. A new distribution metric for image segmentation. Georgia Institute of Technology.

Schuckers, S.A.C., N.A. Schmid, A. Abhyankar, V. Dorairaj and C.K. Boyce et al., 2007. On techniques for angle compensation in nonideal iris recognition. IEEE Trans. Syst. Man Cybernet. Part B, 37: 1176-1190. DOI: 10.1109/TSMCB.2007.904831

Yuan, G.C., Y.J. Liu, M.F. Dion, M.D. Slack and L.F. Wu, 2005. Genome-scale identification of nucleosome positions in S. Cerevisiae. Science, 309: 626-630. DOI: $10.1126 /$ science. 1112178

Zuo, J. and N.A. Schmid, 2010. On a methodology for robust segmentation of nonideal iris images. IEEE Trans. Syst. Man Cybernet. Part B, 40: 703-718. DOI: 10.1109/TSMCB.2009.2015426 